\title{
Constructing Control System Abstractions from Modular Components ${ }^{*}$
}

\author{
Eric S. Kim \\ Department of Electrical Engineering \\ and Computer Sciences, University of \\ California, Berkeley \\ USA \\ eskim@eecs.berkeley.edu
}

\author{
Murat Arcak \\ Department of Electrical Engineering \\ and Computer Sciences, University of \\ California, Berkeley \\ USA \\ arcak@eecs.berkeley.edu
}

\author{
Majid Zamani \\ Department of Electrical and \\ Computer Engineering, Technical \\ University of Munich \\ Germany \\ zamani@tum.de
}

\begin{abstract}
This paper tackles the problem of constructing finite abstractions for formal controller synthesis with high dimensional systems. We develop a theory of abstraction for discrete time nonlinear systems that are equipped with variables acting as interfaces for other systems. Systems interact via an interconnection map which constrains the value of system interface variables. An abstraction of a high dimensional interconnected system is obtained by composing subsystem abstractions with an abstraction of the interconnection. System abstractions are modular in the sense that they can be rearranged, substituted, or reused in configurations that were unknown during the time of abstraction. Constructing the abstraction of the interconnection map can become computationally infeasible when there are many systems. We introduce intermediate variables which break the interconnection and the abstraction procedure apart into smaller problems. Examples showcase the abstraction of a 24-dimensional system through the composition of 24 individual systems, and the synthesis of a controller for a 6-dimensional system with a consensus objective.
\end{abstract}

\section{CCS CONCEPTS}

- Theory of computation $\rightarrow$ Abstraction; - Computer systems organization $\rightarrow$ Embedded and cyber-physical systems; - Networks $\rightarrow$ Formal specifications;

\section{KEYWORDS}

Finite abstractions, Compositionality, Interconnected systems

\section{ACM Reference Format:}

Eric S. Kim, Murat Arcak, and Majid Zamani. 2018. Constructing Control System Abstractions from Modular Components. In Proceedings of 21st International Conference on Hybrid Systems: Computation and Control (part of CPS Week), Porto, Portugal, April 11-13, 2018 (HSCC '18), 10 pages. https://doi.org/10.1145/3178126.3178144

*This work was supported in part by NSF grants CNS-1446145 and ECCS-1405413 and the German Research Foundation (DFG) through the grant ZA 873/1-1.

Permission to make digital or hard copies of all or part of this work for personal or classroom use is granted without fee provided that copies are not made or distributed for profit or commercial advantage and that copies bear this notice and the full citation on the first page. Copyrights for components of this work owned by others than the author(s) must be honored. Abstracting with credit is permitted. To copy otherwise, or republish, to post on servers or to redistribute to lists, requires prior specific permission and/or a fee. Request permissions from permissions@acm.org.

HSCC '18, April 11-13, 2018, Porto, Portugal

() 2018 Copyright held by the owner/author(s). Publication rights licensed to Association for Computing Machinery.

ACM ISBN 978-1-4503-5642-8/18/04 . \$ $\$ 15.00$

https://doi.org/10.1145/3178126.3178144

\section{INTRODUCTION}

In the last decade many techniques have been developed on automated synthesis of controllers enforcing complex logic specifications over complex dynamical systems. Example specifications include properties expressed as linear temporal logic (LTL) formulae or automata on infinite strings [2]. A common approach for providing such controllers is through finite models of concrete dynamical systems. These finite models are constructed such that a discrete controller designed automatically to enforce some properties over the finite model can be refined to a hybrid controller enforcing the same properties over the original concrete system. Finite abstraction approaches include results on piecewise-affine and multi-affine systems $[3,9]$, sound abstractions based on convexity of reachable sets [16], non-uniform abstractions of nonlinear systems over a finite-time horizon [22], complete abstractions for incrementally stable systems [7, 14, 25], sound abstractions for incrementally forward complete systems [26], feedback refinement relations [17], robustness margins [11], mixed-monotone systems [6], and finally complete abstractions for incrementally stable systems without state-space discretization [24].

All the aforementioned results above take a monolithic view of systems, where the entire system is modeled and abstracted. The abstraction-based approach scales poorly when applied monolithically because its complexity grows exponentially in the number of state variables in the model due to the gridding of state sets. As a result, those techniques generally run out of time or memory when confronted with state spaces beyond 4 dimensions.

The scalability challenge can be addressed by constructing finite abstractions using a "split $\rightarrow$ map $\rightarrow$ reduce" pipeline, in that a system is split into smaller interacting components, each component is mapped into an abstraction, and the abstractions are finally combined back into a single monolithic abstraction. The paper's organization reflects this pipeline, but in reverse. Each stage contains a different computational bottleneck, which can be mitigated by previous stages in the pipeline.

Reducing (Section 3) Our main contribution is developing a theoretical foundation that enables one to compose abstractions without depending on structural properties of the concrete system and the interconnection. Internal inputs and outputs act as interfaces between control systems and we provide a notion of alternating simulation functions, which can be used to establish approximate alternating simulation relations from finite systems to concrete ones. Systems interact via an interconnection map that constrains the values of internal variables across systems. Interconnections are also abstracted into discrete approximations satisfying 
the newly introduced notion of an approximate interconnection relation. We provide a compositional result showing that there exists an approximate alternating simulation relation from the interconnection of discrete systems to that of the concrete ones. The only assumption is well posed-ness of the interconnection and a local matching constraint where the abstraction granularity between each system and the interconnection are compatible. This enables a fully modular approach to constructing abstractions by allowing components to be rearranged, substituted, or reused.

Mapping (Section 4) A finite abstraction is computed for each subsystem. The abstraction step can be executed concurrently and independently because each subsystem's internal state is isolated from other systems by the input-output interfaces. The sparsityaware abstraction procedure from [8] can dramatically reduce the abstraction time of systems with sparse dependencies by selectively traversing low-dimensional sub-spaces.

Splitting (Section 5) Unfortunately, sparsity is not always readily apparent to the abstraction algorithm, especially when individual components are viewed as black boxes. We show that it is possible to inject sparsity into interconnections by adding latent variables which capture intermediate computations, thus reducing the complexity of the abstraction step. For instance, any dense matrix representing a linear interconnection can be decomposed into a cascade of sparse matrices via this method.

One example showcases the compositionality of the proposed approach by constructing abstractions of up to $N=24$ systems. The second example showcases controller synthesis for a collection of 6 bimodal systems and a controller that stabilizes around a consensus region.

Related Work. Compositional reasoning approaches for the construction of finite abstractions are developed in [4, 10, 12, 15, 23]. The results in $[15,23]$ use small-gain type conditions to facilitate the compositional construction of complete abstractions. As a result, those techniques require some stability or stabilizability assumptions over multiple subsystems and compositionality conditions can be violated as the number of subsystems increases. In contrast to those results, we provide a compositional construction of sound abstractions without requiring any stability or stabilizability assumption on the subsystems or any implicit condition on the number of subsystems.

The results in [4] and [10] are tailored, respectively, to fully actuated subsystems with globally bounded vector fields and cascade decompositions that arise from single-input partially feedback linearizable systems. On the contrary, our results can be applied to general classes of subsystems modeled as difference inclusions and interconnections modeled as set-valued maps. Our approach is closely related to the one in [12]. Both results provide compositional construction of sound abstractions for interconnected systems modeled as difference inclusions without imposing any compositionality condition. In comparison to our framework, [12] constructs abstractions that 1) are more conservative because the interaction between subsystems are modeled as disturbances whereas our abstractions constrain the interaction with an interconnection map and, 2) require access to the control synthesis specification and the interconnection topology during the abstraction step, whereas we do not require this knowledge.

\section{NOTATION AND PRELIMINARIES}

\subsection{Notation}

The identity map on a set $A$ is denoted by $1_{A}$. If $A$ is a subset of $B$ we denote by $l_{A}: A \hookrightarrow B$ or simply by $\imath$ the natural inclusion map taking any $a \in A$ to $l(a)=a \in B$. We denote by $\mathbb{R}, \mathbb{Z}$, and $\mathbb{N}$ the set of real numbers, integers, and non-negative integers, respectively. These symbols are annotated with subscripts to restrict them in the obvious way, e.g., $\mathbb{Z}_{>0}$ denotes the positive integer numbers. For $a, b \in \mathbb{N}$ and $a \leq b$, we use $[a ; b],(a ; b),[a ; b)$, and $(a ; b]$ to denote the closed, open and half-open intervals in $\mathbb{N}$. For $a, b \in \mathbb{R}$ and $a \leq b$, we use $[a, b],(a, b),[a, b)$, and $(a, b]$ to denote the same intervals in $\mathbb{R}$. Given $N \in \mathbb{N}_{\geq 1}$, vectors $v_{i} \in \mathbb{R}^{n_{i}}$, $n_{i} \in \mathbb{N}_{\geq 1}$, and $i \in[1 ; N]$, we use $v=\left[v_{1} ; \ldots ; v_{N}\right]$ to denote the vector in $\mathbb{R}^{n}$ with $n=\sum_{i=1}^{N} n_{i}$ consisting of the concatenation of vectors $v_{i}$. In $\mathbb{R}^{n}$, the relations $<, \leq, \geq$, > are defined componentwise, e.g., $a<b$ iff $a_{i}<b_{i}$ for all $i \in[1 ; n]$. We denote by $1_{n}$ a vector in $\mathbb{R}^{n}$ with all of its elements to be one. Given a set $A$ and matrix $P$ of appropriate dimension, $P A:=\{P a \mid a \in A\}$. For any $x \in \mathbb{R}^{n}$, we denote by $\|x\|$ the infinity norm of $x$ and by $|x|$ the component-wise absolute value. For any $M \in \mathbb{R}^{n \times m},\|M\|$ denotes the infinity norm of $M$. The closed ball centered at $x \in \mathbb{R}^{n}$ with radius $\varepsilon$ is defined by $\mathcal{B}_{\varepsilon}(x)=\left\{y \in \mathbb{R}^{n} \mid\|x-y\| \leq \varepsilon\right\}$. For any set $A \subseteq \mathbb{R}^{n}$ of the form of finite union of boxes, e.g., $A=\bigcup_{j=1}^{N} A_{j}$ for some $N \in \mathbb{N}$, where $A_{j}=\prod_{i=1}^{n}\left[c_{i}^{j}, d_{i}^{j}\right] \subseteq \mathbb{R}^{n}$ with $c_{i}^{j}<d_{i}^{j}$, and grid parameter $\eta=\left[\eta_{1} ; \ldots ; \eta_{n}\right] \in\left(\mathbb{R}_{>0}\right)^{n}$, where $\eta \leq \operatorname{span}(A)$ and $\operatorname{span}(A)=\left[\min _{j=1, \ldots, N}\left|d_{1}^{j}-c_{1}^{j}\right| ; \ldots ; \min _{j=1, \ldots, N}\left|d_{n}^{j}-c_{n}^{j}\right|\right]$, define $[A]_{\eta}=\bigcup_{j=1}^{N}\left[A_{j}\right]_{\eta}$, where $\left[A_{j}\right]_{\eta}=\left\{a \in A_{j} \mid a_{i}=k_{i} \eta_{i}, k_{i} \in \mathbb{Z}, i=\right.$ $1, \ldots, n\}$. The set $[A]_{\eta}$ will be used as a finite approximation of the set $A$ with precision $\eta$. Note that $[A]_{\eta} \neq \varnothing$ for any $\eta \leq \operatorname{span}(A)$. We denote by $|\cdot|$ the cardinality of a given set and by $\varnothing$ the empty set. If $\eta \in \mathbb{R}_{\geq 0}$ is a scalar, then $[A]_{\eta}=[A]_{\eta 1_{n}}$. We use notations $\mathcal{K}$ and $\mathcal{K}_{\infty}$ to denote the different classes of comparison functions, as follows: $\mathcal{K}=\left\{\alpha: \mathbb{R}_{\geq 0} \rightarrow \mathbb{R}_{\geq 0} \mid \alpha\right.$ is continuous, strictly increasing, and $\alpha(0)=0\} ; \mathcal{K}_{\infty}=\left\{\alpha \in \mathcal{K} \mid \lim _{r \rightarrow \infty} \alpha(r)=\infty\right\}$.

\subsection{Discrete-time control systems}

In this paper we study discrete-time control systems which distinguish inputs and outputs as either internal or external. Internal variables are interfaces to other systems, while external variables are interfaces for controllers.

Definition 2.1. A discrete-time control system $\Sigma$ is defined by the tuple

$$
\Sigma=\left(\mathbb{X}, \mathbb{U}, \mathbb{W}, f, \mathbb{Y}_{1}, \mathbb{Y}_{2}, h_{1}, h_{2}\right)
$$

where $\mathbb{X}, \mathbb{U}, \mathbb{W}, \mathbb{Y}_{1}$, and $\mathbb{Y}_{2}$ are the state set, external input set, internal input set, external output set, and internal output set, respectively, and are assumed to be subsets of normed vector spaces with appropriate finite dimensions. The set-valued map $f: \mathbb{X} \times \mathbb{U} \times \mathbb{W} \rightrightarrows$ $\mathbb{X}$ is called the transition function [18], $h_{1}: \mathbb{X} \rightarrow \mathbb{Y}_{1}$ is the external output map, and $h_{2}: \mathbb{X} \rightarrow \mathbb{Y}_{2}$ is the internal output map. The discrete-time control system $\Sigma$ is described by difference inclusions 
of the form

$$
\Sigma:\left\{\begin{aligned}
x[k+1] & \in f(x[k], u[k], w[k]) \\
y_{1}[k] & =h_{1}(x[k]) \\
y_{2}[k] & =h_{2}(x[k]),
\end{aligned}\right.
$$

where $x[k] \in \mathbb{X}, y_{1}[k] \in \mathbb{Y}_{1}, y_{2}[k] \in \mathbb{Y}_{2}, u[k] \in \mathbb{U}$, and $w[k] \in \mathbb{W}$ are the state, external output, internal output, external input, and internal input at time $k \in \mathbb{N}$.

System $\Sigma=\left(\mathbb{X}, \mathbb{U}, \mathbb{W}, f, \mathbb{Y}_{1}, \mathbb{Y}_{2}, h_{1}, h_{2}\right)$ is called deterministic if $|f(x, u, w)| \leq 1 \forall x \in \mathbb{X}, \forall u \in \mathbb{U}, \forall w \in \mathbb{W}$, and non-deterministic otherwise. System $\Sigma$ is called finite if $\mathbb{X}, \mathbb{U}$, and $\mathbb{W}$ are finite sets and infinite otherwise.

REMARK 1. If $\Sigma$ does not have internal inputs and outputs, Definition 2.1 reduces to the tuple $\Sigma=(\mathbb{X}, \mathbb{U}, f, \mathbb{Y}, h)$ and the set-valued map $f$ becomes $f: \mathbb{X} \times \mathbb{U} \rightrightarrows \mathbb{X}$. Correspondingly, equation (2) reduces to:

$$
\Sigma:\left\{\begin{aligned}
x[k+1] & \in f(x[k], u[k]) \\
y[k] & =h(x[k]) .
\end{aligned}\right.
$$

We use this notion of system to refer later to a monolithic control system constructed from interconnected components.

\subsection{Alternating simulation functions}

We define alternating simulation functions which relate systems $\Sigma$ and $\hat{\Sigma}$ as in Definition 2.1 and quantify the error between them.

Definition 2.2. Consider systems

$$
\Sigma=\left(\mathbb{X}, \mathbb{U}, \mathbb{W}, f, \mathbb{Y}_{1}, \mathbb{Y}_{2}, h_{1}, h_{2}\right),
$$

and

$$
\hat{\Sigma}=\left(\hat{\mathbb{X}}, \hat{\mathbb{U}}, \hat{\mathbb{W}}, \hat{f}, \hat{\mathbb{Y}}_{1}, \hat{\mathbb{Y}}_{2}, \hat{h}_{1}, \hat{h}_{2}\right),
$$

where $\hat{\mathbb{W}} \subseteq \mathbb{W}, \hat{\mathbb{Y}}_{2} \subseteq \mathbb{Y}_{2}$, and $\hat{\mathbb{Y}}_{1}=\mathbb{Y}_{1}$. A function $V: \mathbb{X} \times \hat{\mathbb{X}} \rightarrow \mathbb{R}_{\geq 0}$ is called an alternating simulation function from $\hat{\Sigma}$ to $\Sigma$ if there exist $\alpha_{1}, \alpha_{2} \in \mathcal{K}_{\infty}, \epsilon \in \mathbb{R}_{\geq 0}$, and $\mu \in\left(\mathbb{R}_{>0}\right)^{\operatorname{dim}(\mathbb{W})}$ such that $\forall x \in \mathbb{X}$ and $\forall \hat{x} \in \hat{\mathbb{X}}$ one has

$$
\begin{aligned}
& \alpha_{1}\left(\left\|h_{1}(x)-\hat{h}_{1}(\hat{x})\right\|\right) \leq V(x, \hat{x}), \\
& \alpha_{2}\left(\left\|h_{2}(x)-\hat{h}_{2}(\hat{x})\right\|\right) \leq V(x, \hat{x}),
\end{aligned}
$$

and $\forall(x, \hat{x}) \in\{(x, \hat{x}) \in \mathbb{X} \times \hat{\mathbb{X}} \mid V(x, \hat{x}) \leq \epsilon\}, \forall w \in \mathbb{W}, \forall \hat{w} \in \hat{\mathbb{W}}$ such that $|w-\hat{w}| \leq \mu / 2$, and $\forall \hat{u} \in \hat{\mathbb{U}}, \exists u \in \mathbb{U}, \forall x_{d} \in f(x, u, w)$, $\exists \hat{x}_{d} \in \hat{f}(\hat{x}, \hat{u}, \hat{w})$ such that one gets

$$
V\left(x_{d}, \hat{x}_{d}\right) \leq \epsilon .
$$

When such a $V$ exists, system $\hat{\Sigma}$ is called an abstraction of $\Sigma$.

Note that $\hat{\Sigma}$ may be finite or infinite depending on cardinalities of sets $\hat{\mathbb{X}}, \hat{U}$, and $\hat{\mathbb{W}}$. In the absence of internal inputs and outputs, Definition 2.2 reduces to the following definition.

Definition 2.3. Consider systems $\Sigma=(\mathbb{X}, \mathbb{U}, f, \mathbb{Y}, h)$ and $\hat{\Sigma}=$ $(\hat{\mathbb{X}}, \hat{\mathbb{U}}, \hat{f}, \hat{\mathbb{Y}}, \hat{h})$. A function $V: \mathbb{X} \times \hat{\mathbb{X}} \rightarrow \mathbb{R}_{\geq 0}$ is called an alternating simulation function from $\hat{\Sigma}$ to $\Sigma$ if there exist $\alpha \in \mathcal{K}_{\infty}$ and $\epsilon \in \mathbb{R}_{\geq 0}$ such that $\forall x \in \mathbb{X}$ and $\forall \hat{x} \in \hat{\mathbb{X}}$ one has

$$
\alpha(\|h(x)-\hat{h}(\hat{x})\|) \leq V(x, \hat{x}),
$$

and $\forall(x, \hat{x}) \in\{(x, \hat{x}) \in \mathbb{X} \times \hat{\mathbb{X}} \mid V(x, \hat{x}) \leq \epsilon\}, \forall \hat{u} \in \hat{\mathbb{U}}, \exists u \in \mathbb{U}$, $\forall x_{d} \in f(x, u), \exists \hat{x}_{d} \in \hat{f}(\hat{x}, \hat{u})$ such that one gets

$$
V\left(x_{d}, \hat{x}_{d}\right) \leq \epsilon .
$$

We say that a system $\hat{\Sigma}$ is approximately alternatingly simulated by a system $\Sigma$ or a system $\Sigma$ approximately alternatingly simulates a system $\hat{\Sigma}$, denoted by $\hat{\Sigma} \leq \mathcal{A S} \Sigma$, if there exists an alternating simulation function from $\hat{\Sigma}$ to $\Sigma$ as in Definitions 2.2 and 2.3. When $\hat{\Sigma} \leq \mathcal{A S S} \quad \Sigma$, a controller designed for $\hat{\Sigma}$ can be refined into a controller for the original system $\Sigma$ [21].

REMARK 2. Definition 2.3 implies that the relation $R \subseteq \mathbb{X} \times \hat{\mathbb{X}}$ defined by $R=\{(x, \hat{x}) \in \mathbb{X} \times \hat{\mathbb{X}} \mid V(x, \hat{x}) \leq \epsilon\}$ is an $\varepsilon$-approximate alternating simulation relation, defined in [21], from $\hat{\Sigma}$ to $\Sigma$ with $\varepsilon=\alpha^{-1}(\epsilon)$.

\section{COMPOSITIONAL ABSTRACTIONS FOR INTERCONNECTIONS}

In this section, we analyze networks of discrete-time control systems and show how to construct an alternating simulation function from a network of their abstractions to the concrete network by using alternating simulation functions of the subsystems. The definition of the network of discrete-time control systems is inspired by the notion of interconnected systems in [1].

\subsection{Interconnected systems}

First, we introduce a general notion of interconnection containing the one described in [1].

Definition 3.1. An interconnection $\mathcal{I}$ is simply defined by the tuple $\mathcal{I}=\left(\prod_{i=1}^{N} \mathbb{Y}_{2 i}, \prod_{i=1}^{N} \mathbb{W}_{i}, M\right)$ where $M$ is the set-valued map $M: \prod_{i=1}^{N} \mathbb{Y}_{2 i} \rightrightarrows \prod_{i=1}^{N} \mathbb{W}_{i}$ that maps nondeterministically from internal output values to internal input values.

Given a concrete interconnection $\mathcal{I}$, we introduce here a notion of abstract interconnection $\hat{\mathcal{I}}$ which is used later to construct abstractions of interconnected systems.

Definition 3.2. Consider two interconnections $\mathcal{I}=(\mathbb{Y}, \mathbb{W}, M)$ and $\hat{\mathcal{I}}=(\hat{\mathbb{Y}}, \hat{\mathbb{W}}, \hat{M})$ where $\mathbb{Y}=\prod_{i=1}^{N} \mathbb{Y}_{2 i}$ and $\mathbb{W}=\prod_{i=1}^{N} \mathbb{W}_{i}$. Interconnection $\hat{\mathcal{I}}$ is called an $\left(\epsilon_{y}, \epsilon_{w}\right)$-approximation of $\mathcal{I}$, where $\epsilon_{y} \in\left(\mathbb{R}_{>0}\right)^{\operatorname{dim}(\mathbb{Y})}$ and $\epsilon_{w} \in\left(\mathbb{R}_{>0}\right)^{\operatorname{dim}(\mathbb{W})}$, if $\forall(y, \hat{y}) \in\{(y, \hat{y}) \in$ $\left.\mathbb{Y} \times \hat{\mathbb{Y}}|| y-\hat{y} \mid \leq \epsilon_{y}\right\}, \forall w \in M(y) \exists \hat{w} \in \hat{M}(\hat{y})$ such that $|w-\hat{w}| \leq \epsilon_{w}$. All inequalities are element-wise.

Example 3.3. Consider interconnection $\mathcal{I}=\left(\mathbb{R}^{m}, \mathbb{R}^{p}, M\right)$, where $M \in \mathbb{R}^{p \times m}$ is a matrix. Define the map $\hat{M}: \mathbb{R}^{m} \rightrightarrows\left[\mathbb{R}^{p}\right]_{\mu}$, where $\mu \in\left(\mathbb{R}_{>0}\right)^{p}$, as follows: $\hat{w} \in \hat{M}(\hat{y})$ if $|\hat{w}-M \hat{y}| \leq \mu$. Interconnection $\hat{\mathcal{I}}=\left(\mathbb{R}^{m},\left[\mathbb{R}^{p}\right]_{\mu}, \hat{M}\right)$ is an $\left(\epsilon_{y}, \epsilon_{w}\right)$-approximation of $\mathcal{I}$ if $|M| \epsilon_{y}+$ $\epsilon_{w} \leq \mu$ where $|M|$ is defined as $(|M|)_{i j}=\left|M_{i j}\right|$.

Here, we define the interconnected discrete-time control system as the following.

Definition 3.4. Consider $N \in \mathbb{N}_{\geq 1}$ control subsystems $\Sigma_{i}=$ $\left(\mathbb{X}_{i}, \mathbb{U}_{i}, \mathbb{W}_{i}, f_{i}, \mathbb{Y}_{1 i}, \mathbb{Y}_{2 i}, h_{1 i}, h_{2 i}\right), i \in[1 ; N]$, and an interconnection $\mathcal{I}=\left(\prod_{i=1}^{N} \mathbb{Y}_{2 i}, \prod_{i=1}^{N} \mathbb{W}_{i}, M\right)$ defining the coupling of these subsystems. The interconnected system $\Sigma=(\mathbb{X}, \mathbb{U}, f, \mathbb{Y}, h)$, denoted by $\mathcal{I}\left(\Sigma_{1}, \ldots, \Sigma_{N}\right)$, follows by $\mathbb{X}=\prod_{i=1}^{N} \mathbb{X}_{i}, \mathbb{U}=\prod_{i=1}^{N} \mathbb{U}_{i}, \mathbb{Y}=$ $\prod_{i=1}^{N} \mathbb{Y}_{1 i}$, and maps

$$
\begin{aligned}
f(x, u) & :=\left\{\left[x_{1}^{\prime} ; \ldots ; x_{N}^{\prime}\right] \mid x_{i}^{\prime} \in f_{i}\left(x_{i}, u_{i}, w_{i}\right) \forall i \in[1 ; N]\right\}, \\
h(x) & :=\left[h_{11}\left(x_{1}\right) ; \ldots ; h_{1 N}\left(x_{N}\right)\right],
\end{aligned}
$$


where $u=\left[u_{1} ; \ldots ; u_{N}\right], x=\left[x_{1} ; \ldots ; x_{N}\right]$ and with the internal variables constrained by

$$
\left[w_{1} ; \ldots ; w_{N}\right] \in M\left(\left[h_{21}\left(x_{1}\right) ; \ldots ; h_{2 N}\left(x_{N}\right)\right]\right) .
$$

Internal inputs and outputs allow one to disentangle complex system interactions and construct abstractions for each system individually. Consider a collection of three systems $\Sigma_{1}, \Sigma_{2}, \Sigma_{3}$ where $\Sigma_{1}$ has dynamics $f_{1}\left(x_{1}, M_{1}\left(x_{2}, x_{3}\right)\right)$. By imposing the interconnection constraint $w_{1} \in M_{1}\left(x_{2}, x_{3}\right)$, an equivalent representation is $f_{1}\left(x_{1}, w_{1}\right)$ which only depends on variables local to $\Sigma_{1}$. By disentangling $\Sigma_{1}$ from its dependence on $\Sigma_{2}$ and $\Sigma_{3}$, its abstraction can be computed individually. Furthermore, a different representation $f_{1}\left(x_{1}, x_{2}, x_{3}\right)$ would not reveal that $f_{1}$ 's dependence on $x_{2}$ and $x_{3}$ is via a lower dimensional variable $w_{1}$.

\subsection{Compositionality result}

We assume that we are given $N$ control subsystems

$$
\Sigma_{i}=\left(\mathbb{X}_{i}, \mathbb{U}_{i}, \mathbb{W}_{i}, f_{i}, \mathbb{Y}_{1 i}, \mathbb{Y}_{2 i}, h_{1 i}, h_{2 i}\right),
$$

together with their abstractions

$$
\hat{\Sigma}_{i}=\left(\hat{\mathbb{X}}_{i}, \hat{\mathbb{U}}_{i}, \hat{\mathbb{W}}_{i}, \hat{f}_{i}, \hat{\mathbb{Y}}_{1 i}, \hat{\mathbb{Y}}_{2 i}, \hat{h}_{1 i}, \hat{h}_{2 i}\right),
$$

and alternating simulation functions $V_{i}$ from $\hat{\Sigma}_{i}$ to $\Sigma_{i}$. We use $\alpha_{1 i}$, $\alpha_{2 i}, \epsilon_{i}$, and $\mu_{i}$ to denote the corresponding functions and constants appearing in Definition 2.2.

The next theorem provides a compositional approach on the construction of abstractions of networks of control subsystems and that of the corresponding alternating simulation function.

Theorem 3.5. Consider the interconnected control system $\Sigma=$ $\mathcal{I}\left(\Sigma_{1}, \ldots, \Sigma_{N}\right)$ induced by $N \in \mathbb{N}_{\geq 1}$ subsystems $\Sigma_{i}$ and interconnection $\mathcal{I}=\left(\prod_{i=1}^{N} \mathbb{Y}_{2 i}, \prod_{i=1}^{N} \mathbb{W}_{i}, M\right)$. Suppose that each control subsystem $\Sigma_{i}$ admits an abstraction $\hat{\Sigma}_{i}$ with the corresponding alternating simulation function $V_{i}$ and $\mathcal{I}$ admits an $\left(\epsilon_{y}, \epsilon_{w}\right)$-approximation $\hat{\mathcal{I}}=\left(\prod_{i=1}^{N} \hat{\mathbb{Y}}_{2 i}, \prod_{i=1}^{N} \hat{\mathbb{W}}_{i}, \hat{M}\right)$ with constraints

$$
\begin{aligned}
\epsilon_{y} & =\left[\alpha_{21}^{-1}\left(\epsilon_{1}\right) \mathbf{1}_{\operatorname{dim}\left(\mathbb{Y}_{21}\right)} ; \ldots ; \alpha_{2 N}^{-1}\left(\epsilon_{N}\right) 1_{\operatorname{dim}\left(\mathbb{Y}_{2 N}\right)}\right] \\
\epsilon_{w} & =\left[\mu_{1} / 2 ; \ldots ; \mu_{N} / 2\right]
\end{aligned}
$$

on the internal variables. Let $\epsilon=\max _{i \in[1 ; N]} \epsilon_{i}$. Then function

$$
V(x, \hat{x}):=\max \left\{\frac{\epsilon}{\epsilon_{1}} V_{1}\left(x_{1}, \hat{x}_{1}\right), \ldots, \frac{\epsilon}{\epsilon_{N}} V_{N}\left(x_{N}, \hat{x}_{N}\right)\right\},
$$

is an alternating simulation function, as in Definition 2.3, from abstract interconnected system $\hat{\Sigma}=\hat{\mathcal{I}}\left(\hat{\Sigma}_{1}, \ldots, \hat{\Sigma}_{N}\right)$ to $\Sigma$.

Proof. First we show that inequality (7) holds for some $\mathcal{K}_{\infty}$ function $\alpha$. For any $x=\left[x_{1} ; \ldots ; x_{N}\right] \in \mathbb{X}$ and $\hat{x}=\left[\hat{x}_{1} ; \ldots ; \hat{x}_{N}\right] \in \hat{\mathbb{X}}$ and using (4) for each pair of subsystems $\Sigma_{i}$ and $\hat{\Sigma}_{i}$ and noting that $\frac{\epsilon}{\epsilon_{i}} \geq 1$ for all $i \in[1 ; N]$, one gets:

$$
\begin{aligned}
\|h(x)-\hat{h}(\hat{x})\| & =\left\|\left[h_{11}\left(x_{1}\right) ; \ldots ; h_{1 N}\left(x_{N}\right)\right]-\left[\hat{h}_{11}\left(\hat{x}_{1}\right) ; \ldots ; \hat{h}_{1 N}\left(\hat{x}_{N}\right)\right]\right\| \\
& =\max _{i \in[1 ; N]}\left\|h_{1 i}\left(x_{i}\right)-\hat{h}_{1 i}\left(\hat{x}_{i}\right)\right\| \leq \max _{i \in[1 ; N]} \alpha_{1 i}^{-1}\left(V_{i}\left(x_{i}, \hat{x}_{i}\right)\right) \\
& \leq \bar{\alpha}\left(\max _{i \in[1 ; N]} V_{i}\left(x_{i}, \hat{x}_{i}\right)\right) \\
& \leq \bar{\alpha}\left(\max _{i \in[1 ; N]} \frac{\epsilon}{\epsilon_{i}} V_{i}\left(x_{i}, \hat{x}_{i}\right)\right) \\
& =\bar{\alpha}(V(x, \hat{x})),
\end{aligned}
$$

where $\bar{\alpha}(r)=\max _{i \in[1 ; N]} \alpha_{1 i}^{-1}(r)$ which is a $\mathcal{K}_{\infty}$ function. By defining the $\mathcal{K}_{\infty}$ function $\alpha=\bar{\alpha}^{-1}$, one obtains

$$
\alpha(\|h(x)-\hat{h}(\hat{x})\|) \leq V(x, \hat{x}),
$$

satisfying inequality (7). Now we show that inequality (8) holds as well. Consider any $x=\left[x_{1} ; \ldots ; x_{N}\right] \in \mathbb{X}$ and $\hat{x}=\left[\hat{x}_{1} ; \ldots ; \hat{x}_{N}\right] \in \hat{\mathbb{X}}$, where $V(x, \hat{x}) \leq \epsilon$. From equation (13), we conclude that $V_{i}\left(x_{i}, \hat{x}_{i}\right) \leq$ $\epsilon \forall i \in[1 ; N]$. Since $\hat{\mathcal{I}}$ is an $\left(\epsilon_{y}, \epsilon_{w}\right)$-approximation of $\mathcal{I}$ with $\epsilon_{w}=$ $\left[\mu_{1} / 2 ; \ldots ; \mu_{N} / 2\right]$ and $\epsilon_{y}=\left[\alpha_{21}^{-1}(\epsilon) \mathbf{1}_{\operatorname{dim}\left(\mathbb{Y}_{21}\right)} ; \ldots ; \alpha_{2 N}^{-1}(\epsilon) \mathbf{1}_{\operatorname{dim}\left(\mathbb{Y}_{2 N}\right)}\right]$ and

$$
\left|\left[\begin{array}{c}
h_{21}\left(x_{1}\right) \\
\vdots \\
h_{2 N}\left(x_{N}\right)
\end{array}\right]-\left[\begin{array}{c}
\hat{h}_{21}\left(\hat{x}_{1}\right) \\
\vdots \\
\hat{h}_{2 N}\left(\hat{x}_{N}\right)
\end{array}\right]\right| \leq\left[\begin{array}{c}
\alpha_{21}^{-1}(\epsilon) \mathbf{1}_{\operatorname{dim}\left(\mathbb{Y}_{21}\right)} \\
\vdots \\
\alpha_{2 N}^{-1}(\epsilon) \mathbf{1}_{\operatorname{dim}\left(\mathbb{Y}_{2 N}\right)}
\end{array}\right],
$$

then for any $\left[w_{1} ; \ldots ; w_{N}\right] \in M\left(\left[h_{21}\left(x_{1}\right) ; \ldots ; h_{2 N}\left(x_{N}\right)\right]\right)$ there exists $\left[\hat{w}_{1} ; \ldots ; \hat{w}_{N}\right] \in \hat{M}\left(\left[\hat{h}_{21}\left(\hat{x}_{1}\right) ; \ldots ; \hat{h}_{2 N}\left(\hat{x}_{N}\right)\right]\right)$ such that the following vector inequality holds

$$
\left|\left[\begin{array}{c}
w_{1} \\
\vdots \\
w_{N}
\end{array}\right]-\left[\begin{array}{c}
\hat{w}_{1} \\
\vdots \\
\hat{w}_{N}
\end{array}\right]\right| \leq\left[\begin{array}{c}
\mu_{1} / 2 \\
\vdots \\
\mu_{N} / 2
\end{array}\right]
$$

Hence, we conclude that $\left|w_{i}-\hat{w}_{i}\right| \leq \mu_{i} / 2$ for all $i \in[1 ; N]$, required for condition (6) to hold for each pair of subsystems $\Sigma_{i}$ and $\hat{\Sigma}_{i}$. Consider any $\hat{u}=\left[\hat{u}_{1} ; \ldots ; \hat{u}_{N}\right] \in \hat{\mathbb{U}}$. Using Definition 2.2, for any $i \in[1 ; N]$, there exists $u_{i} \in \mathbb{U}_{i}$, consequently, a vector $u=\left[u_{1} ; \ldots ; u_{N}\right] \in \mathbb{U}$ such that for any $x_{d} \in f(x, u)$ there exists $\hat{x}_{d} \in \hat{f}(\hat{x}, \hat{u})$ satisfying (6) for each pair of subsystems $\Sigma_{i}$ and $\hat{\Sigma}_{i}$. Finally, by having (6) for each pair of subsystems $\Sigma_{i}$ and $\hat{\Sigma}_{i}$ and using (13), we obtain $V\left(x_{d}, \hat{x}_{d}\right) \leq \epsilon$ which concludes the proof.

Theorem 3.5 allows one to construct a finite abstraction of a network of subsystems using finite abstractions of subsystems as depicted in Figure 1. This abstraction satisfies Definition 2.3, which ensures that behavior that is enforced on $\hat{\Sigma}$ can also be approximately enforced on $\Sigma$. The only compatibility condition between systems $\hat{\Sigma}_{i}$ and the interconnection $\hat{\mathcal{I}}$ are (12) and (11). These conditions are both component-wise so modifying or replacing one system $\hat{\Sigma}_{i}$ does not affect the compatibility condition between $\hat{\mathcal{I}}$ and another system $\hat{\Sigma}_{j}$.

\section{CONSTRUCTION OF FINITE ABSTRACTIONS}

In this section, we consider $\Sigma$ as an infinite control system and $\hat{\Sigma}$ as its finite abstraction. Now, we propose a way of constructing a finite abstraction $\hat{\Sigma}$ of $\Sigma$.

Definition 4.1. Let $\Sigma=\left(\mathbb{X}, \mathbb{U}, \mathbb{W}, f, \mathbb{X}, \mathbb{X}, 1_{\mathbb{X}}, 1_{\mathbb{X}}\right)$ be a control system, where $\mathbb{X}, \mathbb{W}$ are assumed to be finite unions of boxes and $\mathbb{U}$ is a finite set. One can construct a finite system

$$
\hat{\Sigma}=\left(\hat{\mathbb{X}}, \hat{\mathbb{U}}, \hat{\mathbb{W}}, \hat{f}, \hat{\mathbb{Y}}_{1}, \hat{\mathbb{Y}}_{2}, \hat{h}_{1}, \hat{h}_{2}\right) \text {, }
$$

where:

(1) $\hat{\mathbb{X}}=[\mathbb{X}]_{\eta}$ where $\eta \in \mathbb{R}_{\geq 0}$ is a state set quantization parameter satisfying the vector inequality $0<\eta 1_{\operatorname{dim}(\mathbb{X})} \leq \operatorname{span}(\mathbb{X})$.

(2) $\hat{\mathbb{U}}=\mathbb{U}$ 


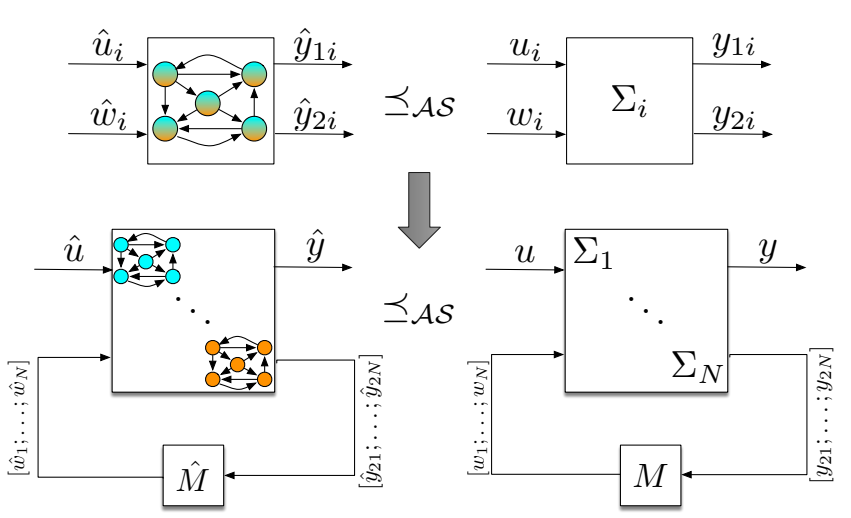

Figure 1: Compositionality result under when $\hat{\Sigma}_{i}$ are finite systems. A collection of abstractions $\hat{\Sigma}_{i}$ (above arrow) can be interconnected while still satisfying the alternating simulation relation $\leq \mathcal{A} S$

(3) $\hat{\mathbb{W}}=[\mathbb{W}]_{\mu}$, where $\mu \in \mathbb{R}_{\geq 0}$ is the internal input set quantization parameter satisfying the vector inequality $0<\mu \mathbf{1}_{\operatorname{dim}(\mathbb{W})} \leq$ $\operatorname{span}(\mathbb{W})$.

(4) $\hat{x}_{d} \in \hat{f}(\hat{x}, \hat{u}, \hat{w})$ if $^{1} \mathcal{B}_{\eta / 2}\left(\hat{x}_{d}\right) \cap f\left(\mathcal{B}_{\eta / 2}(\hat{x}), \hat{u}, \mathcal{B}_{\mu / 2}(\hat{w})\right) \neq \varnothing$

(5) $\hat{\mathbb{Y}}_{1}=\mathbb{X}, \hat{\mathbb{Y}}_{2}=\hat{\mathbb{X}}, \hat{h}_{1}=\imath: \hat{\mathbb{X}} \hookrightarrow \hat{\mathbb{Y}}_{1}$ and $\hat{h}_{2}=1_{\hat{\mathbb{X}}}$.

Now, we present the main result of this section establishing the relation between $\Sigma$ and $\hat{\Sigma}$, introduced above, via the notion of alternating simulation function.

THEOREM 4.2. Let $\Sigma$ be an infinite control system and $\hat{\Sigma}$ be its corresponding discrete system as in Definition 4.1. Then $V(x, \hat{x})=$ $\|x-\hat{x}\|, \forall x \in \mathbb{X}, \forall \hat{x} \in \hat{\mathbb{X}}$, is an alternating simulation function from $\hat{\Sigma}$ to $\sum$.

Proof. It can be readily verified that function $V$ satisfies conditions (4) and (5) with functions $\alpha_{1}=\alpha_{2}=1_{\mathbb{R}_{\geq 0}}$. Now we show that $V$ also satisfies condition (6) by $\epsilon=\|\eta\| / 2$. Consider any $x \in \mathbb{X}$ and $\hat{x} \in \hat{\mathbb{X}}$ where $|x-\hat{x}| \leq \eta / 2$ along with any $w \in \mathbb{W}$ and $\hat{w} \in \hat{\mathbb{W}}$ where $|w-\hat{w}| \leq \mu / 2$. For any $\hat{u} \in \hat{\mathbb{U}}$ we may choose input $u=\hat{u} \in \mathbb{U}$. Now, consider any $x_{d} \in f(x, u, w)$ in $\Sigma$. Since $\mathbb{X}$ is a finite union of boxes, there exists $\hat{x}_{d} \in \hat{\mathbb{X}}$ such that:

$$
\left|x_{d}-\hat{x}_{d}\right| \leq \eta / 2 \text {. }
$$

Moreover we readily obtain $\mathcal{B}_{\eta / 2}\left(\hat{x}_{d}\right) \cap f\left(\mathcal{B}_{\eta / 2}(\hat{x}), \hat{u}, \mathcal{B}_{\mu / 2}(\hat{w})\right) \neq$ $\varnothing$ which, by definition of $\hat{\Sigma}$, implies the existence of $\hat{x}_{d} \in \hat{f}(\hat{x}, \hat{u}, \hat{w})$ in $\hat{\Sigma}$. Therefore, from inequality (15) and since $\|\eta\| / 2=\epsilon$, we can conclude that condition (6) in Definition 2.2 holds which completes the proof.

Using a similar line of reasoning, one can construct a finite abstraction $\hat{\mathcal{I}}$ of the interconnection $\mathcal{I}$.

Definition 4.3. Let $\mathcal{I}=(\mathbb{Y}, \mathbb{W}, M)$ be an interconnection where $\mathbb{Y}$ is a finite union of boxes. One can construct an interconnection $\hat{\mathcal{I}}=(\hat{Y}, \hat{\mathbb{W}}, \hat{M})$ that is an $\left(\epsilon_{y}, \epsilon_{w}\right)$-approximation as follows:

\footnotetext{
${ }^{1}$ Here, the notation $f\left(\mathcal{B}_{\eta / 2}(\hat{x}), \hat{u}, \mathcal{B}_{\mu / 2}(\hat{w})\right)$ means an over-approximation of the set $\bigcup_{x \in \mathcal{B}_{\eta / 2}(\hat{x})} \bigcup_{w \in \mathcal{B}_{\mu / 2}(\hat{w})} f(x, \hat{u}, w)$.
}

(1) $\hat{\mathbb{Y}}=[\mathbb{Y}]_{\epsilon_{y}}$

(2) $\hat{\mathbb{W}}=[\mathbb{W}]_{\epsilon_{w}}$

(3) $\hat{w} \in \hat{M}(\hat{y})$ if $^{2} \mathcal{B}_{\epsilon_{w} / 2}(\hat{w}) \cap M\left(\mathcal{B}_{\epsilon_{y} / 2}(\hat{y})\right) \neq \varnothing$.

\subsection{Efficient Representations of Abstractions}

Because the abstract systems $\hat{\Sigma}_{i}$ are over finite state domains, it is possible to view the transition relation $\hat{f}$ as a finite state automaton or graph. The efficiency of constructing, storing, and manipulating these abstractions depends on the underlying data structure used to represent the system. Although it is possible to store the automaton as a lookup table or a sparse matrix, these data structures require memory that is inherently exponential with respect to the state space dimension. Composing systems as defined in Definition 3.4 is also prohibitively expensive for these system representations because the number of discrete states grows exponentially.

The storage and manipulation problems are mitigated by representing the system and interconnection abstractions with binary decision diagrams (BDD) [5], a data structure that stores abstractions implicitly as Boolean-valued functions. These functions represent system dynamics by returning true whenever a transition $\left(\hat{x}, \hat{u}, \hat{w}, \hat{x}_{d}\right)$ is valid for system $\hat{\Sigma}$. For interconnections $\hat{\mathcal{I}}=(\hat{U}, \hat{Y}, \hat{M})$, the function returns true when $(\hat{u}, \hat{y})$ satisfies the relation $\hat{y} \in \hat{M}(\hat{u})$. BDDs are a symbolic representation and often exhibit a smaller memory footprint compared to explicit representations that store every transition in memory. We use the CUDD toolbox [20] for BDDs, which provides functionality for common BDD operations such as taking conjunctions, disjunctions, or existential/universal quantification over a set of variables. Composing and interconnecting systems is achieved through Boolean conjunctions over all constraints (9) and (10) specified in Definition 3.4. Existing tools SCOTS[19] and CoSyMa[13] take control systems and construct symbolic representations by systematically ensuring that Item 4 in Definition 4.1 holds.

\subsection{Leveraging Sparsity for Constructing Abstractions}

Regardless of the system representation, constructing an abstraction monolithically often requires a brute force traversal of the space $\hat{\mathbb{X}} \times \hat{U} \times \hat{\mathbb{W}}$ unless $f$ exhibits some favourable structural properties. The number of operations required for this brute force traversal suffers from an exponential blowup with respect to the dimension of $\hat{\mathbb{X}} \times \hat{U} \times \hat{\mathbb{W}}$.

One useful structural property for system abstraction is sparsity, where the evolution of one state depends only on a few variables. These dependencies among different system components allow one to reduce the runtime of the system abstraction because the brute force search occurs over lower dimensional subspaces.

As an illustrative example consider a concrete system $\Sigma_{i}$ with a three dimensional state space $\mathbb{X}_{i}=\mathbb{X}_{i}^{1} \times \mathbb{X}_{i}^{2} \times \mathbb{X}_{i}^{3}$, a scalar valued internal input $\mathbb{W}_{i}=\mathbb{W}_{i}^{1}$, no external input, and with dynamics $f_{i}$

\footnotetext{
${ }^{2}$ Here, the notation $M\left(\mathcal{B}_{\epsilon y / 2}(\hat{y})\right)$ means an over-approximation of the set $\bigcup_{y \in \mathcal{B}_{\epsilon} / 2}(\hat{y}) M(y)$.
} 
that can be written component-wise as:

$$
\begin{aligned}
x_{i}^{1}[k+1] & =f_{i}^{1}\left(x_{i}^{1}[k], w_{i}^{1}[k]\right) \\
x_{i}^{2}[k+1] & =f_{i}^{2}\left(x_{i}^{2}[k], x_{i}^{3}[k]\right) \\
x_{i}^{3}[k+1] & =f_{i}^{3}\left(x_{i}^{3}[k], x_{i}^{1}[k]\right) .
\end{aligned}
$$

Each state update depends on two variables. A naive brute force approach for abstracting $f_{i}$ would traverse a four dimensional grid $\hat{\mathbb{X}}_{i}^{1} \times \hat{\mathbb{X}}_{i}^{2} \times \hat{\mathbb{X}}_{i}^{3} \times \hat{\mathbb{W}}_{i}^{1}$ and compute a three dimensional set that contains ${ }^{3}$ the value $x_{i}[k+1]$. A more efficient approach is to traverse three lower dimensional spaces and compute over-approximations of $x_{i}^{1}[k+1], x_{i}^{2}[k+1], x_{i}^{3}[k+1]$ individually. In order for the abstraction algorithm to know which low dimensional spaces to traverse, it needs a user-provided hint in the form of a dependency graph. The dependency graph for the aforementioned example is given below with $x_{i}^{j}[k+1]$ represented by $x_{i}^{j+}$. For each next state vertex $x_{i}^{j+}$ the indegree represents the dimensionality of the lower dimensional space traversal.

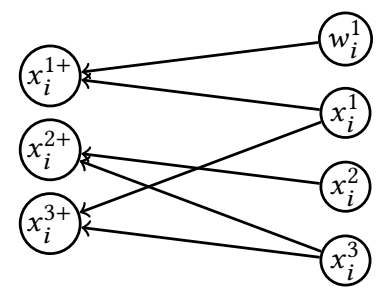

By adopting a functional view of systems $f_{i}: \mathbb{X}_{i} \times \mathbb{U}_{i} \times \mathbb{W}_{i} \rightrightarrows \mathbb{X}_{i}$ and interconnections $M: \mathbb{Y} \rightrightarrows \mathbb{W}$, discrete abstractions of both can be computed with Algorithm 1 which presents pseudo code for an algorithm that computes symbolic abstractions of generic, possibly set valued, functions. Initially the abstraction $\hat{f}$ contains no constraints on the output. The algorithm computes an abstraction $\hat{f}_{i}$ that constrains the $i$-th output of $f$. It takes advantage of the BDD representation of $\hat{f}_{i}$ and combines them with a logical AND in Line 6. The number of operations for computing abstraction $\hat{f}: \hat{A} \rightarrow \hat{B}$ is linear with respect to $\operatorname{dim}(B)$ and exponential with respect to the maximum indegree of the dependency graph, $\max _{i} \operatorname{dim}\left(D\left(b_{i}\right)\right)$, due to the lower-dimensional grid traversal in Line 5.

The idea to incorporate dependency structures for abstracting monolithic systems was formalized in [8], which did not include the notion of internal variables or interconnections. The following section describes a methodology to expose additional sparsity in interconnections.

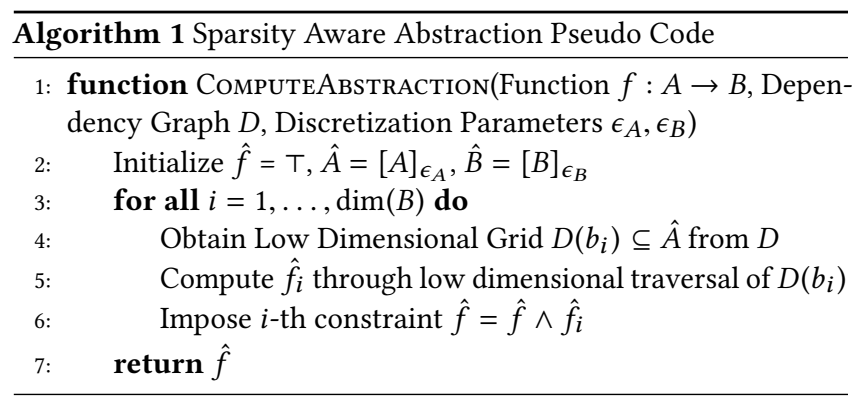

${ }^{3}$ Recall that reachable set over-approximations are used in Definition 4.1 and Definition 4.3

\section{ABSTRACTION OF DECOMPOSABLE INTERCONNECTIONS}

Systems are interconnected by imposing constraints on internal input and output variables as in (10). A symbolic representation of the interconnection must also be constructed to create an abstract interconnected system. Construction of arbitrary interconnections is in the worst case exponential with respect to the number of systems $N$. In this section we show how additional variables representing intermediate computations can introduce sparsity and reduce the computational burden of abstraction.

Assumption 1. For illustrative purposes and notational simplicity, we assume in this section that the interconnection is a linear map represented with matrix $M$ and each $w_{i}$ and $y_{2 i}$ are scalar real values for all $i=1, \ldots, N$. Only minor modifications are required in the vector valued case and the nonlinear case is addressed in Remark 3.

Abstracting the interconnection matrix $M$ incurs the maximum computation cost when all elements of a row are nonzero. Consider the following interconnection matrix for $N=4$

$$
M=\left[\begin{array}{llll}
1 & 1 & 1 & 1 \\
0 & 0 & 0 & 1 \\
0 & 0 & 0 & 1 \\
0 & 0 & 0 & 1
\end{array}\right] .
$$

The top row signifies that a single internal input $w_{1}$ is influenced by all internal outputs, while the right column signifies that one internal output $y_{2 N}$ influences all internal inputs. Computing $\hat{w}_{1}$ as a function of $\hat{y}_{21}, \ldots, \hat{y}_{2 N}$ is exponential with respect to $N$ because Algorithm 1 needs to query every value of $\hat{y}_{2 i}$. This corresponds to the monolithic interconnection depicted schematcially in Figure 2, where an internal output variable's influence on $w_{1}$ depends on the other values.

We present a method to reduce this exponent for interconnections that can be decomposed into smaller parts. In reality, the top row of $M$ is simply a weighted addition of $N$ numbers which can easily be broken apart if additional auxiliary variables are introduced to represent intermediate sums. Figure 3 and Figure 4 reveal that the originally monolithic interconnection from Figure 2 contains hidden structural properties that can be exploited by introducing intermediate variables $z_{1}$ and $z_{2}$. The original $M$ with a dense row can be decomposed into a product of matrices, each with at most two non-zero entries per row as seen below. Labels are provided to give intuition for the roles of each column and row.

$$
M=w_{1} \begin{array}{ccccccc}
y_{21} & y_{22} & y_{23} & y_{24} & z_{1} & z_{2} \\
w_{3} & w_{4}
\end{array}\left[\begin{array}{cccccc}
0 & 0 & 0 & 0 & 1 & 1 \\
0 & 0 & 0 & 1 & 0 & 0 \\
0 & 0 & 0 & 1 & 0 & 0 \\
0 & 0 & 0 & 1 & 0 & 0
\end{array}\right]\left[\begin{array}{ccccc}
1 & 0 & 0 & 0 \\
0 & 1 & 0 & 0 \\
0 & 0 & 1 & 0 \\
0 & 0 & 0 & 1 \\
1 & 1 & 0 & 0 \\
0 & 0 & 1 & 1
\end{array}\right]
$$

\subsection{Exposing Matrix Sparsity with Intermediate Variables}

We formalize a method to generate a sparse matrix decomposition by first leveraging the notion of a dependency graph for interconnections. 

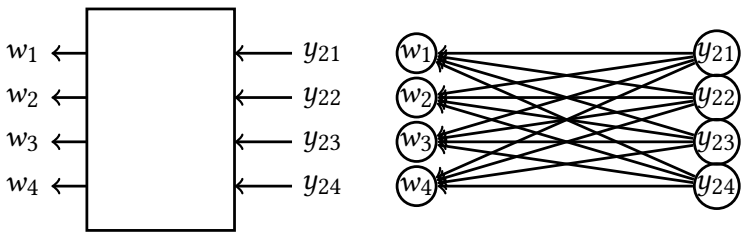

Figure 2: Monolithic view of interconnection $M$ in (16) as a black box, where all internal inputs $w_{i}$ may depend on all internal outputs $y_{2} j$ in the associated dependency graph.
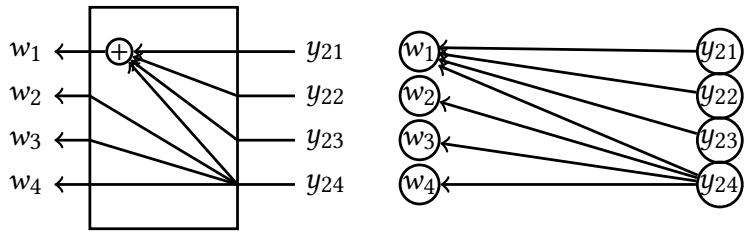

Figure 3: Interconnection with dependencies exposed. The dependency graph exhibits a reduced number of edges, but $w_{1}$ still exhibits a high indegree and requires traversing a 4-dimensional grid to construct an abstraction.
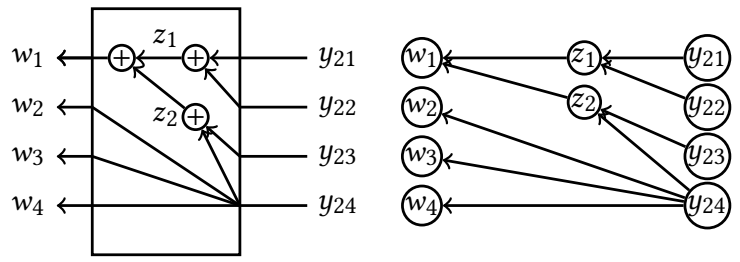

Figure 4: Adding intermediate vertices $z_{1}, z_{2}$ to the graph reduces the maximum indegree from 4 to 2 .

Definition 5.1. An interconnection dependency graph is a directed acyclic graph $D=(V, E)$ where each vertex represents a variable and

(1) internal inputs and outputs are a subset of the set of vertices

$$
\left\{w_{1}, \ldots, w_{N}, y_{21}, \ldots, y_{2 N}\right\} \subseteq V ;
$$

(2) There is no path from $w_{i}$ to $w_{j}$ for any $i, j \in\{1, \ldots, N\}$;

(3) There is no path from $y_{2 i}$ to $y_{2 j}$ for any $i, j \in\{1, \ldots, N\}$;

(4) There is no path from $w_{j}$ to $y_{2 i}$ for any $i, j \in\{1, \ldots, N\}$;

(5) Each $w_{i}$ is reachable from at least one $y_{2 j}$ for any $i, j \in$ $\{1, \ldots, N\}$.

The length of a path is the number of edges it contains. The depth of the graph is the longest path from any internal input vertex to any internal output vertex. Note that there may be additional vertices in the graph that are not associated with an internal input or output. A dependency graph is said to be minimal if

$$
V=\left\{w_{1}, \ldots, w_{N}, y_{21}, \ldots, y_{2 N}\right\} .
$$

Dependency graphs for interconnections associated with matrices can be augmented with additional information on each edge which contains the matrix coefficients $M_{i j}$. We can assign a minimal graph to any matrix $M \in \mathbb{R}^{N \times N}$ by placing an edge $\left(y_{2 i}, w_{j}\right)$ from $y_{2 i}$ to $w_{j}$ with coefficient $c\left(y_{2 i}, w_{j}\right)=M_{j i}$ if and only if $M_{j i} \neq 0$. Given any desired indegree $D \in\{2, \ldots, N\}$, a new dependency graph can be constructed by adding intermediate vertices as shown in the next lemma.

Lemma 5.2. For each matrix $M \in \mathbb{R}^{L \times N}$, there exists a corresponding dependency graph where each vertex has an indegree $<D$.

Proof. Let $D_{M}=\left(V_{M}, E_{M}\right)$ be the minimal dependency graph of $M$. If all internal input vertices have an indegree at most $D$, then the assertion is trivially satisfied. Suppose otherwise, and let $w_{i}$ be an internal input vertex with indegree $s_{i}>D$. Add $\left\lceil\frac{s_{i}-D}{D-1}\right\rceil$ intermediate vertices (for example $z_{1}, z_{2}$ in Figure 4 ). There are initially $s_{i}$ "unconsumed" internal output vertices. Each intermediate vertex consumes $D$ unconsumed variables and produces one intermediate unconsumed variable that represents their (possibly weighted) sum. After using all $\left\lceil\frac{s_{i}-D}{D-1}\right\rceil$ intermediate vertices, less than $D$ unconsumed vertices remain and an edge from each to $w_{i}$ is added. This process is repeated for each internal input vertex with indegree greater than $D$.

The main role of Lemma 5.2 is to demonstrate the existence of a sparse dependency graph from an arbitrary matrix $M$. It does not specify how the additional intermediate vertices should be added and interconnected. Many different augmented dependency graphs can be created.

Suppose a dependency graph of the interconnection $M$ is given such that it contains internal input vertices with indegree $>D$. It is possible to reduce the indegrees by decomposing $M$ into a product of sparse matrices each with no more than $D$ non-zeros per row.

Proposition 5.3. For interconnection matrix $M$ let $s$ be the depth of any valid dependency graph $D=(V, E)$ and $k=|V|-N$. There exist matrices $L_{1}, \ldots, L_{s}$ such that

$$
M=\prod_{i=1}^{s} L_{i}=L_{s} \ldots L_{1}
$$

where each $L_{i}$ has at most $D$ non-zero entries on every row, $L_{s} \in$ $\mathbb{R}^{N \times k}, L_{i} \in \mathbb{R}^{k \times k}$ for all $i=2, \ldots, s-1$, and $L_{1} \in \mathbb{R}^{k \times N}$.

Proof. By definition $k$ is the number of vertices that do not correspond to internal inputs $w_{1}, \ldots, w_{N}$. Let $g=|V|-2 N$ and $\left\{z_{1}, \ldots, z_{g}\right\}$ be the set of variables (or vertices of $D$ ) that are neither internal inputs nor outputs. For each intermediate vertex $z_{i}$, we associate a value $p\left(z_{i}\right)$ which equals the path length from $z_{i}$ to the furthest internal output vertex. Matrices with sides of length $k$ are associated with the concatenated vector $\left[y_{2}, z\right]$ where $y_{2}=$ $\left[y_{21}, \ldots, y_{2 N}\right] \in \mathbb{R}^{N}$. Define matrices $L_{1}, \ldots, L_{s}$ component-wise such that

$$
\left(L_{1}\right)_{l m}= \begin{cases}1 & \text { if } l=m \\ c\left(y_{m}, z_{l-N}\right) & \text { if } p\left(z_{l-N}\right)=1 \text { and }\left(y_{m}, z_{l-N}\right) \in E \\ 0 & \text { otherwise. }\end{cases}
$$

For all $i=2, \ldots, r-1$

$\left(L_{i}\right)_{l m}=\left\{\begin{array}{l}1 \\ 1 \\ c\left(y_{m}, z_{l-N}\right) \\ c\left(z_{m-N}, z_{l-N}\right) \\ 0\end{array}\right.$

if $l=m \leq N$

if $l=m>N$ and $p\left(z_{l-N}\right)>i$

if $p\left(z_{l-N}\right)=i$ and $\left(y_{m}, z_{l-N}\right) \in E$

if $p\left(z_{l-N}\right)=i$ and $\left(z_{m-N}, z_{l-N}\right) \in E$ otherwise, 
and

$$
\left(L_{s}\right)_{l m}= \begin{cases}c\left(y_{m}, w_{l}\right) & \text { if }\left(y_{m}, w_{l}\right) \in E \\ c\left(z_{m-N}, w_{l}\right) & \text { if }\left(z_{m-N}, w_{l}\right) \in E \\ 0 & \text { otherwise. }\end{cases}
$$

Lemma 5.2 and Proposition 5.3 jointly show that any interconnection matrix $M$ can be decomposed into the composition of a sequence of sparse components and reduce the complexity of constructing the interconnection. While auxiliary variables induce additional non-determinism, but this cost is generally offset by an exponential reduction in abstraction computation time. The maximum number of additional variables is quadratic with respect to $N$ (assuming scalar valued $w_{i}$ and $y_{2 i}$ ). The quadratic dependence arises because there are $N$ internal inputs $w_{i}$, and the maximum number of outputs required by any single $w_{i}$ in the worst case is linear with respect to $N$ as shown in Lemma 5.2's proof.

Remark 3. Proposition 5.3 is specialized for matrices but its decomposition result can easily be generalized whenever the interconnection can be broken apart into smaller operations. If these operations are associative, then the sparse dependency graph is not in general unique because different orders of evaluating internal operations result in different intermediate values. Non-uniqueness is actually a positive feature of associative operations because it provides greater flexibility when constructing new dependency graphs.

The aforementioned results have all focused on decomposing interconnections into sparse components. The following result is analogous to Theorem 3.5 and shows that composing approximate abstractions of interconnections yields an approximate abstraction of the composed concrete interconnections.

TheOREM 5.4. Let $\mathcal{I}=\left(\prod_{i=1}^{N} \mathbb{Y}_{i}, \prod_{i=1}^{N} \mathbb{W}_{i}, M\right)$ have a decomposition such that $M=L_{s} \circ \cdots \circ L_{1}$ where

$$
\begin{aligned}
& \mathcal{I}_{1}=\left(\prod_{i=1}^{N} \mathbb{Y}_{i}, \prod_{i=1}^{N} \mathbb{Y}_{i} \times \mathbb{Z}_{1}, L_{1}\right) \\
& \mathcal{I}_{j}=\left(\prod_{i=1}^{N} \mathbb{Y}_{i} \times \mathbb{Z}_{j}, \prod_{i=1}^{N} \mathbb{Y}_{i} \times \mathbb{Z}_{j+1}, L_{j}\right) \text { for } j=2, \ldots, s-1 \\
& \mathcal{I}_{s}=\left(\prod_{i=1}^{N} \mathbb{Y}_{i} \times \mathbb{Z}_{s}, \prod_{i=1}^{N} \mathbb{W}_{i}, L_{s}\right) .
\end{aligned}
$$

Let each $\hat{\mathcal{I}}_{j}$ (with a corresponding $\left.\hat{L}_{j}\right)$ be an $\left(\epsilon_{j}, \epsilon_{j+1}\right)$ approximation of $\mathcal{I}_{j}$. Then $\hat{\mathcal{I}}$ is an $\left(\epsilon_{1}, \epsilon_{s}\right)$-approximation of $\mathcal{I}$.

Proof. Consider any pair $\left(y_{1}, \hat{y}_{1}\right)$ such that $\left\|y_{1}-\hat{y}_{1}\right\| \leq \epsilon_{1}$. Because $\mathcal{I}_{j}$ is an $\left(\epsilon_{j}, \epsilon_{j+1}\right)$ approximation, for all $y_{j+1} \in L_{j}\left(y_{j}\right)$ there exists a $\hat{y}_{j+1} \in \hat{L}_{j}\left(\hat{y}_{j}\right)$ such that $\left\|y_{j+1}-\hat{y}_{j+1}\right\| \leq \epsilon_{j+1}$. The $\left(y_{j+1}, \hat{y}_{j+1}\right)$ can then be used to apply the definition of $\left(\epsilon_{j+1}, \epsilon_{j+2}\right)$ to obtain a new pair $\left(y_{j+2}, \hat{y}_{j+2}\right)$. Such an argument continues by induction until a pair $\left(y_{s}, \hat{y}_{s}\right)$ satisfying $\left\|y_{s}-\hat{y}_{s}\right\| \leq \epsilon_{s}$ is generated, satisfying the conditions for $\left(\epsilon_{1}, \epsilon_{s}\right)$-approximation given in Definition 3.2.

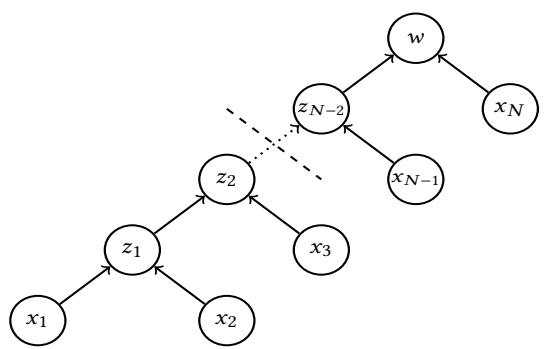

Figure 5: Tree structure for decomposing an $N$-ary max. A cumulative maximum is represented with $N-2$ additional intermediary vertices $z_{1}, \ldots, z_{N-2}$. Each parent node's variable is the maximum of its two children, e.g. $z_{1}=\max \left(x_{1}, x_{2}\right)$.

\section{EXAMPLE}

We use a modified version of the toolbox SCOTS [19] to construct the discrete, symbolic abstractions of the systems from Definition 4.1 and the interconnection from Definition 4.3. The modifications incorporate the sparsity-aware algorithm for system abstraction that appeared in [8] but also generalize it to construct abstractions of interconnections. All systems are represented symbolically as Boolean functions with the CUDD toolbox [20] and all examples are run on a 2013 Macbook Pro with 2.4GHz Intel Core i7 processor and 8GB of RAM.

\subsection{Scalable Construction of Interconnection Abstractions}

The sparsity aware algorithm from [8] is only scalable if each system state is only influenced by a small set of variables. The following system does not satisfy such a sparsity condition because all systems interact with a single global variable. It is designed to illustrate the scalability of the interconnection decompositions in Section 5 over a range of $N$.

Consider a collection of $N$ systems where $\mathbb{X}_{i}=\mathbb{Y}_{i}=[0,31]$, $\mathbb{U}_{i}=[0,7], \mathbb{W}=[0,31]$ and

$$
\begin{aligned}
x_{i}[k+1] & =f_{i}\left(x_{i}[k], u_{i}[k], w[k]\right) \\
& =\min \left(K\left(x_{i}[k]+u_{i}[k]\right), w[k]+1,31\right),
\end{aligned}
$$

where $K=.75$ and there is a single $w[k] \in \mathbb{W}$ shared amongst all subsystems

$$
w[k]=\max \left(x_{1}[k], \ldots, x_{N}[k]\right) .
$$

Substituting Equation (26) into Equation (25) reveals that $x_{i}$ depends on the state of all systems, however, this dependency can be succinctly represented with a single value $w[k]$ that is shared amongst all $N$ systems. Furthermore, by leveraging the following decomposition of the $N$-ary maximum operation,

$$
\begin{aligned}
z_{1} & =\max \left(x_{1}, x_{2}\right) \\
z_{i} & =\max \left(z_{i-1}, x_{i+1}\right) \text { for all } i=2, \ldots, N-2 \\
w & =\max \left(z_{N-2}, x_{N}\right)
\end{aligned}
$$

constructing the abstract interconnection $\hat{\mathcal{I}}=\left(\prod_{i=1}^{N} \hat{\mathbb{X}}_{i}, \mathbb{W}, \hat{M}\right)$ can be done efficiently via the indegree two graph in Figure 5. 


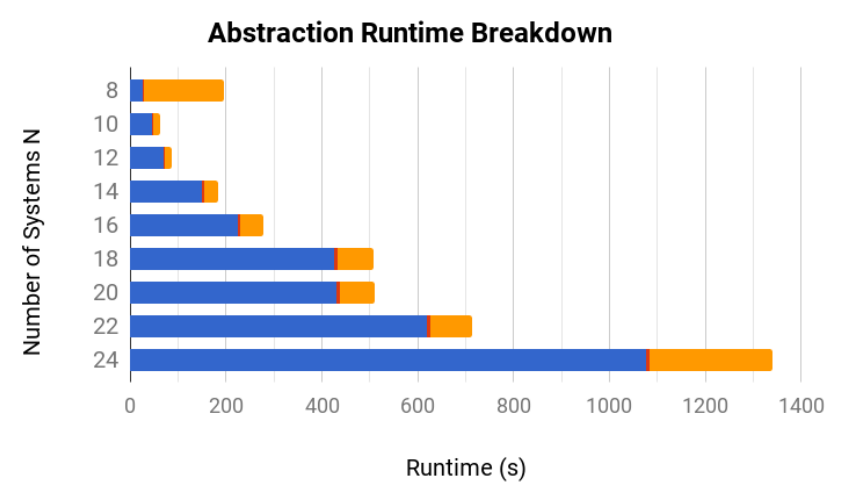

Table 1: Runtimes for systems (25) and interconnection (26) for varying $N$. The cumulative runtime is represented by the entire horizontal bar, which is broken apart as follows: blue (left color) signifies time to abstract and parallel compose all systems, red (middle color) signifies the relatively negligible interconnection construction time, and orange (right color) is the time to compose the system and interconnection abstractions.

The abstraction grids $\hat{\mathbb{X}}_{i}$ and $\hat{\mathbb{W}}$ were constructed with parameters $\eta=\mu=1$ and the external input grid is $\hat{U}=[0,7]_{1}$. Each abstract system has 32 discrete states, 8 external inputs, and 32 internal inputs. Each intermediate variable is identical to $\hat{\mathbb{X}}_{i}$. Table 1 shows the runtimes to construct the set of $N$ abstract systems and the abstract interconnection. Although the number of BDD operations is linear with respect to $N$, each individual operation runtime may vary due to the different BDDs sizes and the overhead from heuristic algorithms in the CUDD package used to minimize the size of BDDs.

\subsection{Consensus Around an Unstable Region for a Collection of Bimodal Systems}

We consider a set of $N$ systems with spaces $\mathbb{X}_{i}=\mathbb{Y}_{i}=[0,31]$, $\mathbb{U}_{i}=[-2,2]$ for each $i=1, \ldots, N$ and a shared internal input $\mathbb{W}=[0,31]$. The dynamics for system $\Sigma_{i}$ are given by

$$
\begin{aligned}
x_{i}[k+1] & =f_{i}\left(x_{i}[k], u_{i}[k], w_{i}[k]\right) \\
& =\operatorname{GLOG}_{[0,31]}\left(x_{i}[k]+u_{i}[k]+K\left(x_{i}[k]-w[k]\right)\right)
\end{aligned}
$$

where $K=0.1$ and $\operatorname{GLOG}_{[0,31]}(\cdot)$ is a generalized logistic function with output values within the range $[0,31]$

$$
\operatorname{GLOG}_{[0,31]}(x)=\frac{31}{1+e^{-.2\left(x-\frac{b+a}{2}\right)}} .
$$

The output function $h_{2 i}: \mathbb{X}_{i} \rightarrow \mathbb{Y}_{i}$ is simply the identity function.

The interconnected system needs to satisfy a stability objective where it must reach and remain within the consensus region:

$$
\phi=\bigvee_{\theta=0,1, \ldots, 31}\left(\bigwedge_{i=1}^{N}\left(\left\|x_{i}-\theta\right\| \leq 3\right)\right),
$$

signifying that all agents have states $x_{i}$ that lie within a neighborhood of a common value $\theta$. After the consensus region is reached,

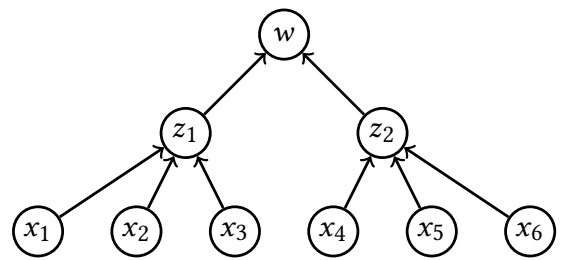

Figure 6: Dependency graph for computing the aggregate average for $N=6$ with low dimensional operations. Note that $y_{i}=x_{i}$.

the value of $\theta$ may still change over time as the interconnected system executes. If $\theta$ were a known and fixed constant then the objective would be trivially decomposable into $N$ pieces and a controller could be synthesized for each subsystem independently. Because $\theta$ may vary and is not fixed beforehand, it is non-trivial to synthesize a controller for each subsystem $\Sigma_{i}$ individually if the controllers only have access to the local state $x_{i}$. The reach and remain objective is succinctly expressed in temporal logic as $\diamond \square \phi$.

Two system properties impede satisfaction of $\diamond \square \phi$. First, for fixed values $u_{i}=0$ and $w=0$, system (28) exhibits an unstable equilibrium around $x_{i}^{*}=15.5$ and two stable equilibira around $x_{i}^{*}=$ 1.92 and $x_{i}^{*}=29.07$. This bimodal property is problematic due to the divergence that occurs when different systems have initial states above and below 15.5. The second difficulty in enforcing $\diamond \square \phi$ is due to the interaction amongst systems. The concrete interconnection $w \in M(x)$ is a deterministic map encoding the average state among all systems.

$$
w=\frac{1}{N} \sum_{i=1}^{N} x_{i}
$$

Note that $w$ is a function of $N$ variables and lies within the region $[0,31]$ for all values of the joint state $x \in \prod_{i=1}^{N} \mathbb{X}_{i}$. If $u_{i}[k]=0$, then the term $K\left(x_{i}-w\right)$ applies an additive term that pushes the state $x_{i}$ away from the average and makes the consensus region $\phi$ inherently unstable.

Exogenous input $w$ depends on $N=6$ values and constructing the interconnection abstraction monolithically is computationally prohibitive. The interconnection is decomposed with the dependency graph depicted in Figure 6. Two intermediate variables $z_{1}, z_{2} \in[0,31]$ represent intermediate averages.

$$
z_{1}=\frac{1}{3}\left(x_{1}+x_{2}+x_{3}\right) \quad \text { and } \quad z_{2}=\frac{1}{3}\left(x_{4}+x_{5}+x_{6}\right) .
$$

With these intermediate variables, the internal inputs are given by

$$
w=\frac{1}{2}\left(z_{1}+z_{2}\right) \text {. }
$$

Substituting the equations in (31) into (32) yields the original interconnection (30) for $N=6$.

Each system is abstracted with state abstraction parameters $\eta=1$, and internal input parameter $\mu=2$, external input grid $\hat{U}=[-2,2]_{1}$. The intermediate variables are discretized such that $\hat{\mathbb{Z}}_{1}, \hat{\mathbb{Z}}_{2}=[0,31]_{2}$. Each discrete system has 32 states, 5 external inputs, and 16 internal inputs. The interconnected abstract system has $1.074 \times 10^{9}$ states and the consensus region $\phi$ has $1.8 \times 10^{6}$ discrete states. Abstracting and parallel composition of the systems 
System Trajectories for Example 6.2

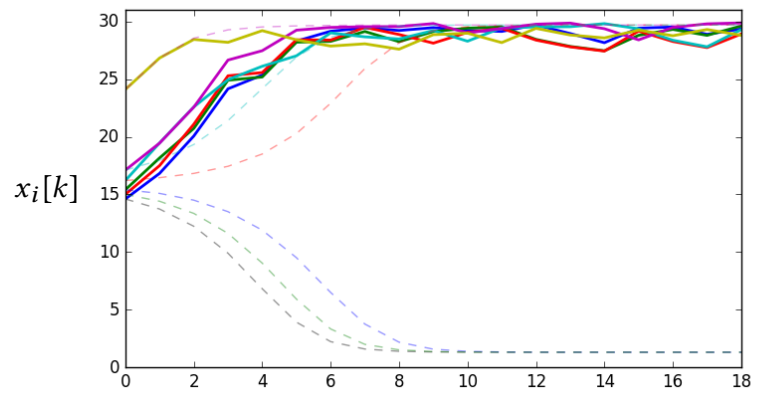

Random Inputs Satisfying $\hat{u}[k] \in C(\hat{x}[k])$

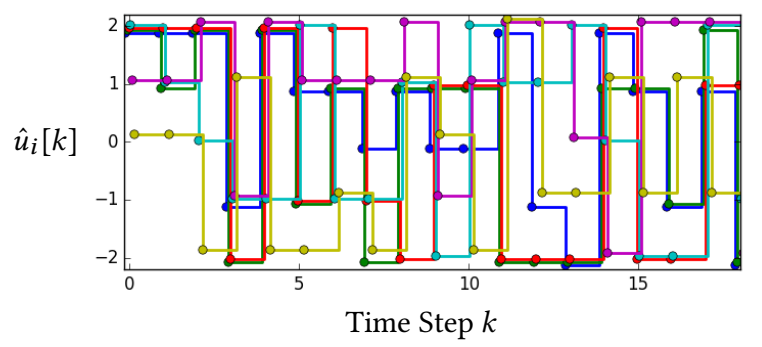

Figure 7: (Top Figure) Two 6-dimensional trajectories depicted as two sets of six scalar valued trajectories. Each line represents a trajectory $x_{i}[\cdot]$ for some $i$. Solid lines correspond to a controller that enforces $\diamond \square \phi$. Dashed lines correspond to an inactive controller with $u_{i}[k]=0$ for all systems $i$ and time steps $k$. Both sets of trajectories have the same initial state $x[0]=[14.6 ; 15.4 ; 15 ; 16.2 ; 17.1 ; 24.1]$ which is outside the consensus region $\phi$. (Bottom Figure) The piecewise constant control inputs to enforce $\diamond \square \phi$, depicted with slight vertical and horizontal perturbations for visual clarity.

took $8.62 s$, constructing the interconnection abstraction took $2.14 \mathrm{~s}$, and synthesis of $\diamond \square \phi$ 's reachable basin with $8.61 \times 10^{8}$ states for the interconnected system took $3702.9 s$. At each state $\hat{x}$, the controller yields a set of allowable external inputs $C(\hat{x}) \subseteq \hat{U}$. As long as $\hat{u}[k] \in C(\hat{x}[k])$ at all time steps then each system will satisfy the specification. Figure 7 depicts two trajectories corresponding to an inactive controller and a controller that, respectively, violate and enforce satisfaction of $\diamond \square \phi$. In Figure 7 control inputs $\hat{u}[k]$ are randomly chosen from $C(\hat{x}[k])$. Attempts to abstract the system with the algorithm in [8] timed out after two hours.

\section{REFERENCES}

[1] M. Arcak, C. Meissen, and A. Packard. 2016. Networks of dissipative systems. Springer International Publishing.

[2] C. Baier and J. P. Katoen. 2008. Principles of model checking. The MIT Press.

[3] C. Belta and L.C.G.J.M. Habets. 2006. Controlling a class of nonlinear systems on rectangles. IEEE Trans. Automat. Control 51, 11 (2006), 1749-1759.

[4] D. Boskos and D. V. Dimarogonas. 2015. Decentralized abstractions for feedback interconnected multi-agent systems. In Proceedings of 54th IEEE Conference on Decision and Control. 282-287.

[5] R. E. Bryant. 1986. Graph-Based Algorithms for Boolean Function Manipulation. IEEE Trans. Comput. 35, 8 (1986), 677-691.

[6] S. Coogan and M. Arcak. 2015. Efficient finite abstraction of mixed monotone systems. In Proceedings of 18th International Conference on Hybrid Systems: Computation and Control. 58-67.
[7] A. Girard, G. Pola, and P. Tabuada. 2010. Approximately bisimilar symbolic models for incrementally stable switched systems. IEEE Trans. Automat. Control 55, 1 (January 2010), 116-126.

[8] F. Gruber, E. Kim, and M. Arcak. 2017. Sparsity-Aware Finite Abstraction. In 2017 IEEE 56th Conference on Decision and Control (CDC).

[9] L.C.G.J.M. Habets, P.J. Collins, and J.H. Van Schuppen. 2006. Reachability and control synthesis for piecewise-affine hybrid systems on simplices. IEEE Trans. Automat. Control 51, 6 (2006), 938-948.

[10] O. Hussein, A. Ames, and P. Tabuada. 2017. Abstracting partially feedback linearizable systems compositionally. IEEE Control Systems Letters 1, 2 (October 2017), 227-232.

[11] J. Liu and N. Ozay. 2014. Abstraction, discretization, and robustness in temporal logic control of dynamical systems. In Proceedings of the 17th International Conference on Hybrid Systems: Computation and Control. ACM New York, NY, USA, 293-302.

[12] P. J. Meyer, A. Girard, and E. Witrant. 2017, accepted. Compositional abstraction and safety synthesis using overlapping symbolic models. IEEE Trans. Automat. Control (2017, accepted).

[13] S. Mouelhi, A. Girard, and G. Gössler. 2013. CoSyMA: A Tool for Controller Synthesis Using Multi-scale Abstractions. In 16th International Conference on Hybrid Systems: Computation and Control. ACM, 83-88.

[14] G. Pola, A. Girard, and P. Tabuada. 2008. Approximately bisimilar symbolic models for nonlinear control systems. Automatica 44, 10 (October 2008), 25082516.

[15] G. Pola, P. Pepe, and M. D. Di Benedetto. 2016. Symbolic models for networks of control systems. IEEE Trans. Automat. Control 61, 11 (November 2016), 3663-3668.

[16] G. Reißig. 2011. Computing abstractions of nonlinear systems. IEEE Transaction on Automatic Control 56, 11 (November 2011), 2583-2598.

[17] G. Reißig, A. Weber, and M. Rungger. April 2017. Feedback Refinement Relations for the Synthesis of Symbolic Controllers. IEEE Trans. Automat. Control 62, 4 (April 2017), 1781-1796.

[18] R. T. Rockafellar and R. Wets. 2009. Variational analysis. Vol. 317. Springer.

[19] M. Rungger and M. Zamani. 2016. SCOTS: A Tool for the Synthesis of Symbolic Controllers. In 19th International Conference on Hybrid Systems: Computation and Control. ACM, 99-104.

[20] F. Somenzi. 2015. CUDD: CU Decision Diagram Package. http://vlsi.colorado. $\mathrm{edu} /$ fabio/CUDD/. (2015). Version 3.0.0.

[21] P. Tabuada. 2009. Verification and Control of Hybrid Systems. New York, NY,USA: Springer.

[22] Y. Tazaki and J. Imura. 2009. Discrete-state abstractions of nonlinear systems using multi-resolution quantizer. In International Conference on Hybrid Systems: Computation and Control (HSCC). Lecture Notes in Computer Science, Vol. 5469. Springer Verlag, Berlin Heidelberg, 351-365.

[23] Y. Tazaki and J. I. Imura. 2008. Bisimilar finite abstractions of interconnected systems. In 11th International Conference on Hybrid Systems: Computation and Control. Springer Berlin Heidelberg, Berlin, Heidelberg, 514-527.

[24] M. Zamani, A. Abate, and A. Girard. 2015. Symbolic models for stochastic switched systems: A discretization and a discretization-free approach. Automatica 55 (May 2015), 183-196.

[25] M. Zamani, P. Mohajerin Esfahani, R. Majumdar, A. Abate, and J. Lygeros. 2014. Symbolic control of stochastic systems via approximately bisimilar finite abstractions. IEEE Trans. Automat. Control 59, 12 (November 2014), 3135-3150.

[26] M. Zamani, G. Pola, M. Mazo Jr., and P. Tabuada. 2012. Symbolic models for nonlinear control systems without stability assumptions. IEEE Transaction on Automatic Control 57, 7 (July 2012), 1804-1809. 